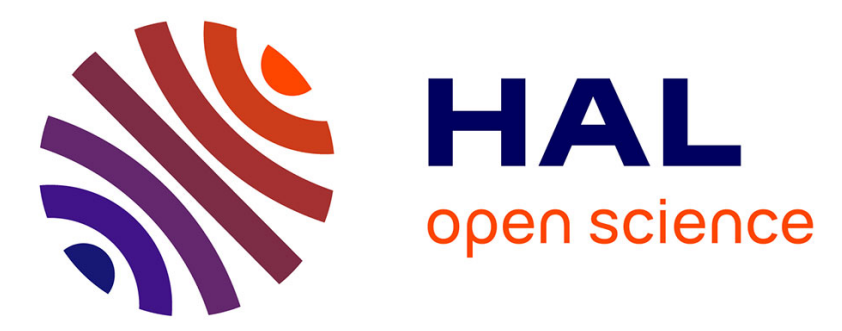

\title{
DISCRIMINATION ACOUSTIQUE DE L'INSUFFISANCE VÉLAIRE CHEZ L'ENFANT
}

F. Plante, C. Berger-Vachon, I. Kauffmann, J. Borel

\section{To cite this version:}

F. Plante, C. Berger-Vachon, I. Kauffmann, J. Borel. DISCRIMINATION ACOUSTIQUE DE L'INSUFFISANCE VÉLAIRE CHEZ L'ENFANT. Journal de Physique IV Proceedings, 1992, 02 (C1), pp.C1-323-C1-326. 10.1051/jp4:1992169 • jpa-00251241

\section{HAL Id: jpa-00251241 https://hal.science/jpa-00251241}

Submitted on 1 Jan 1992

HAL is a multi-disciplinary open access archive for the deposit and dissemination of scientific research documents, whether they are published or not. The documents may come from teaching and research institutions in France or abroad, or from public or private research centers.
L'archive ouverte pluridisciplinaire HAL, est destinée au dépôt et à la diffusion de documents scientifiques de niveau recherche, publiés ou non, émanant des établissements d'enseignement et de recherche français ou étrangers, des laboratoires publics ou privés. 


\title{
DISCRIMINATION ACOUSTIQUE DE LINSUFFISANCE VÉLAIRE CHEZ L'ENFANT
}

\author{
F. PLANTE*, C. BERGER-VACHON ${ }^{*, * *}$, I. KAUFFMANN* et J. BOREL* \\ * Département de Physiologie Sensorielle Audition et Voix (URA CNRS 1447), Pavillon U, Hôpital \\ E. Herriot, 3 place d'Arsonval, F-69437 Lyon cedex 03, France \\ ** Laboratoire d'Electronique de Mesure et d'Instrumentation, Université Claude Bemard, 43 bd du \\ 11 novembre 1918, F-69622 Villeurbanne, France
}

\begin{abstract}
Résumé
Dans cette étude, les auteurs cherchent à discriminer la phonation d'enfants témoins de celle d'enfants insuffísants vélaires. Les voyelles $/ \mathrm{a} / \mathrm{i} / \mathrm{i} /$, et $/ \mathrm{u} / \mathrm{sont}$ étudiées. Les résultats obtenus dans les espaces cepstral et formantique sont comparés.

L'espace cepstral permet une meilleure discrimination. On peut obtenir $100 \%$ de séparation en regroupant les voyelles $/ \mathrm{i} / \mathrm{et} / \mathrm{u} /$. Cependant l'espace formantique permet une interprétation physique. Dans l'espace des deux premiers formants on retrouve les conclusions des études sur la nasalité (cible nasale).
\end{abstract}

\begin{abstract}
In this paper, the authors cxplored the discrimination of two groups of 19 control children and 12 patients with velar impairment. Vowels $/ \mathrm{a} /, / \mathrm{i} /$ and $/ \mathrm{u} /$ had been studied. Results in cepstral space and formantic space were compared.

Cepstral space allows the best discrimination. A $100 \%$ recognition score can be achieved when the two vowels / $\mathrm{i} /$ and $/ \mathrm{u} /$ are considered together. On the other hand formantic space allows a physic interpretation. In the two first formant space nasal target described in the litterature are shower.
\end{abstract}

\section{INTRODUCTION}

Actuellement en pratique médicale courante, l'évaluation des pathologies vocales est réalisée de façon auditive subjective par le médecin ORL. De nombreuses méthodes (endoscopie, électromyographie, aérodynamique, ...) tentent d'objectiver cette évaluation pour permettre une quantification plus précise de la pathologie. Mais beaucoup d'entre elles ont un caractère désagréable, voire invasif, et renseignent plus sur les conditions anatomo-fonctionnelles que sur le résultat phonatoire. L'étude des troubles chez l'enfant pose de façon plus cruciale le problème du caractère invasif. L'analyse acoustique semble donc une méthode non invasive pertinente pour lobjectivation du diagnostic ORL.

Dans cette étude nous nous intéressons aux troubles liés à l'insuffisance vélaire. Le manque de mobilité du voile du palais, qui ne peut plus ainsi réaliser la séparation entre les cavités nasale et buccopharyngée, engendre deux types de perturbations. La première est une nasalisation de la parole, qui fait l'objet de ce travail. La deuxième est une difficulté à former les occlusives à cause de la fuite nasale. L'effet de la nasalisation est étudié sur les voyelles $/ \mathrm{a} /$, /i/ et $/ \mathrm{u} /$.

Sous le terme d'analyse acoustique se regroupent un certain nombre de méthodes qui différent aussi 
bien sur les conditions d'utilisation que sur leurs possibilités. Dans cette étude on cherche des paramètres permettant de séparer la phonation d'enfants atteints d'insuffisance vélaire de celle d'enfants témoins. Cela nécessite de réduire l'information du signal à un certain nombre de paramètres pertinents. Ceci est réalisé par la prédiction linéaire[1]. Mais de nombreux paramètres peuvent être calculés à partir de cette méthode (coefficients de réflexion, cepstraux, d'aire, ...). D'un point de vue théorique, les coefficients cepstraux semblent les meilleurs pour séparer les deux populations car ils permettent de calculer aisément la distance entre deux spectres[2]. Cependant leur interprétation physique est difficile alors que les formants permettent de mieux comprendre les mécanismes mis en jeu. On a donc comparé les résultats de séparation obtenus dans chacun des deux espaces pour évaluer lcur intérêt respectif dans la réalisation d'un diagnostic objectif. L'analyse discriminante est utilisée pour quantifier la séparation des deux populations.

\section{MATERIEL ET METHODES.}

Les populations se composent de 19 témoins, âgés entre 5.5 et 10.4 ans, et de 12 insuffisants vélaires de 5 à 10.7 ans. Tous les enfants ont subi un examen ORL complet.

Les voyelles $/ \mathrm{a} / \mathrm{i} / \mathrm{i} /$ et $/ \mathrm{u} /$ sont enregistrées sur un magnétophone puis digitalisées à une fréquence de $20 \mathrm{KHz}$. A partir de critères visuels, les parties stables des voyelles sont segmentées à l'aide du logiciel IIS (Interactive Laboratory System). La durée de segmentation varie entre 75 et $100 \mathrm{~ms}$.

Une prédiction linéaire glissante d'ordre 18 est effectuée : sur une fenêtre d'analyse de $10 \mathrm{~ms}$ que l'on décale successivement de $5 \mathrm{~ms}$, les coefficients cepstraux et formantiques sont calculés. Les résultats sont ensuite moyennés pour obtenir les jeux de coefficients caractéristiques de la prononciation. 18 coefficients cepstraux et 16 coefficients formantiques ( 8 formants et leur bande passante) sont ainsi calculés. L'analyse discriminante est utilisée pour quantifier la séparation entre les deux populations.

\section{RESULTATS}

Les matrices de confusion et le pouvoir de discrimination (distance entre les deux groupes) obtenus à partir de l'analyse discriminante sont regroupés dans le tableau I. Dans les matrices de confusion, en ligne figure le groupe d'appartenance ( $T$ pour les témoins et I.V pour les insuffisants vélaires), et en colonne le groupe dans lequel l'analyse discriminante classe le sujet.

Pour le /a/ et le /u/ il manque deux sujets dans l'espace formantique car les formants n'ont pu être calculés automatiquement.

Pour l'espace cepstral, la voyelle /i/ est la meilleure (1 seul mal classé, avec le plus grand pouvoir de discrimination). Pour l'espace formantique, la meilleure voyelle est le $/ \mathrm{u} /$. Mais ce résultat doit être relativisé compte tenu de l'absence de deux sujets.

Pour toutes les voyelles on obtient de meilleurs résultats avec les coefficients cepstraux. 


\section{DISCUSSION}

Pour la discrimination il est plus intéressant d'utiliser les coefficients cepstraux. Les voyelles fermées /i/ et / $\mathrm{u} /$ sont les meilleures, mais elles ne permettent pas d'avoir une séparation parfaite. Cependant en positionnant les sujets dans l'espace des axes discriminant $\mathrm{du} / \mathrm{i} / \mathrm{et} / \mathrm{u} /$ (Figure 1) on peut tracer une frontière linéaire entre les deux groupes.

Mais ce résultat ne permet pas d'indiquer les différences phonatoires entre les deux populations. On a donc positionné les sujets dans l'espace vocalique des deux premiers formants (Figure 2). On s'aperçoit que l'on retrouve les résultats connus sur la nasalité [3]. On a bien un déplacement des insuffisants vélaires vers la cible nasale (zone de l'espace (F1-F2) se situant vers $400-1200 \mathrm{~Hz}$ ).

Tableau $1:$ Résultats de l'analyse discriminante.

\begin{tabular}{|c|c|c|c|c|}
\hline \multirow[t]{2}{*}{ Voyelle } & \multicolumn{2}{|c|}{ matrice de confusion } & \multicolumn{2}{|c|}{ pouvoir de discrimination } \\
\hline & cepstraux & formants & cepstraux & formants \\
\hline /a/ & $\begin{array}{ccc}\mathrm{A} \mid \mathrm{C} & \mathrm{T} & \mathrm{I} . \mathrm{V} \\
\mathrm{T} & 18 & 1 \\
\mathrm{I}, \mathrm{V} & 1 & 11\end{array}$ & $\begin{array}{ccc}\mathrm{A} \backslash \mathrm{C} & \mathrm{T} & \mathrm{I} . \mathrm{V} \\
\mathrm{T} & 16 & 3 \\
\mathrm{I} . \mathrm{V} & 1 & 9\end{array}$ & 2.32 & 1.45 \\
\hline ii/ & $\begin{array}{ccc}\mathrm{A} \backslash \mathrm{C} & \mathrm{T} & \mathrm{I} . \mathrm{V} \\
\mathrm{T} & 18 & 1 \\
\mathrm{I.V} & 0 & 12\end{array}$ & $\begin{array}{ccc}\text { AlC } & \text { T } & \text { I.V } \\
T & 17 & 2 \\
\text { I.V } & 1 & 11\end{array}$ & 2.64 & 1.9 \\
\hline$/ \mathrm{u} /$ & $\begin{array}{ccc}\text { AlC } & \mathrm{T} & \mathrm{I} . \mathrm{V} \\
\mathrm{T} & 18 & 1 \\
\mathrm{I} . \mathrm{V} & 1 & 11\end{array}$ & $\begin{array}{ccc}\text { AlC } & \text { T } & \text { I.V } \\
T & 17 & 1 \\
I . V & 1 & 10\end{array}$ & 2.35 & 2.34 \\
\hline
\end{tabular}

\section{CONCLUSION}

Pour obtenir une séparation entre les témoins et les insuffisants vélaires, l'espace cepstral semble plus pertinent que l'espace formantique. Cependant pour une plus grande validité des résultats, il est nécessaire d'intorduire une interprétation physique. Or celle-ci nécessite de travailler dans l'espace formantique.

Pour une étude complète de la pathologie il est difficile de ne retenir qu'un seul espace de représentation. L'étude dans chacun des deux espaces (formantique-cepstral) doit donc se faire en parallèle.

\section{BIBLIOGRAPHIE}

[1] MARKEL J.D., GRAY A.H., Linear Prediction of Speech. New-York, Springer-Verlag, (1976).

[2] GRAY A.H., MARKEL J.D, Distances Measures for Speech Processing. IEEE Trans ASSP, vol.24, p.380 (1976).

[3] DICKSON D., An Acoustic Study of Nasality. J. Speech Hear. Res. vol.5, p.103 (1962).

\section{REMERCIEMENTS}

Cette étude n'aurait pas pu être réalisée sans le soutien de la société API (Audiologie Prothèse Innovation) de Lyon et la région Rhône-Alpes. 


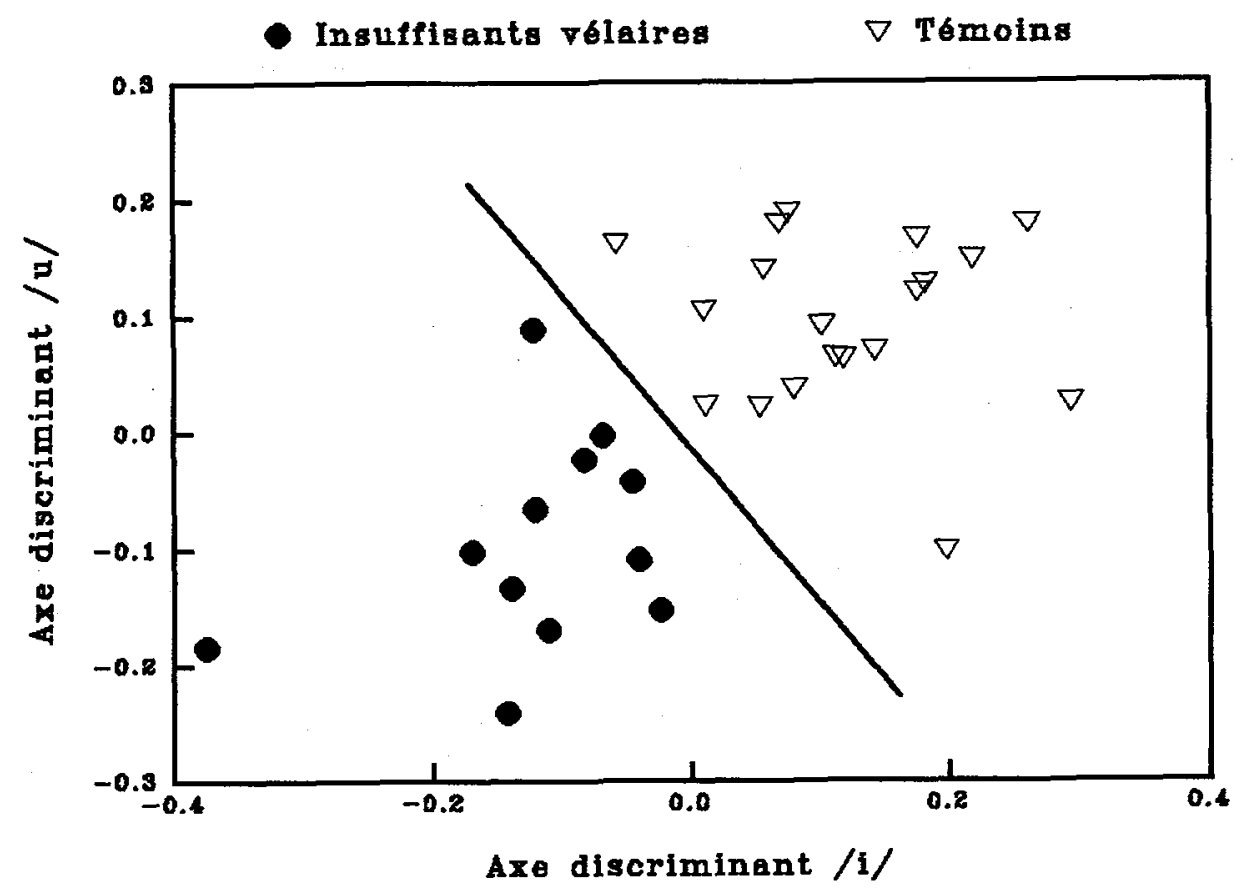

Figure 1 : Sujets dans l'espace des axes discriminants /i/ et / $\mathbf{w} /$.

$$
\text { deuxième formant }(\mathrm{Hz})
$$

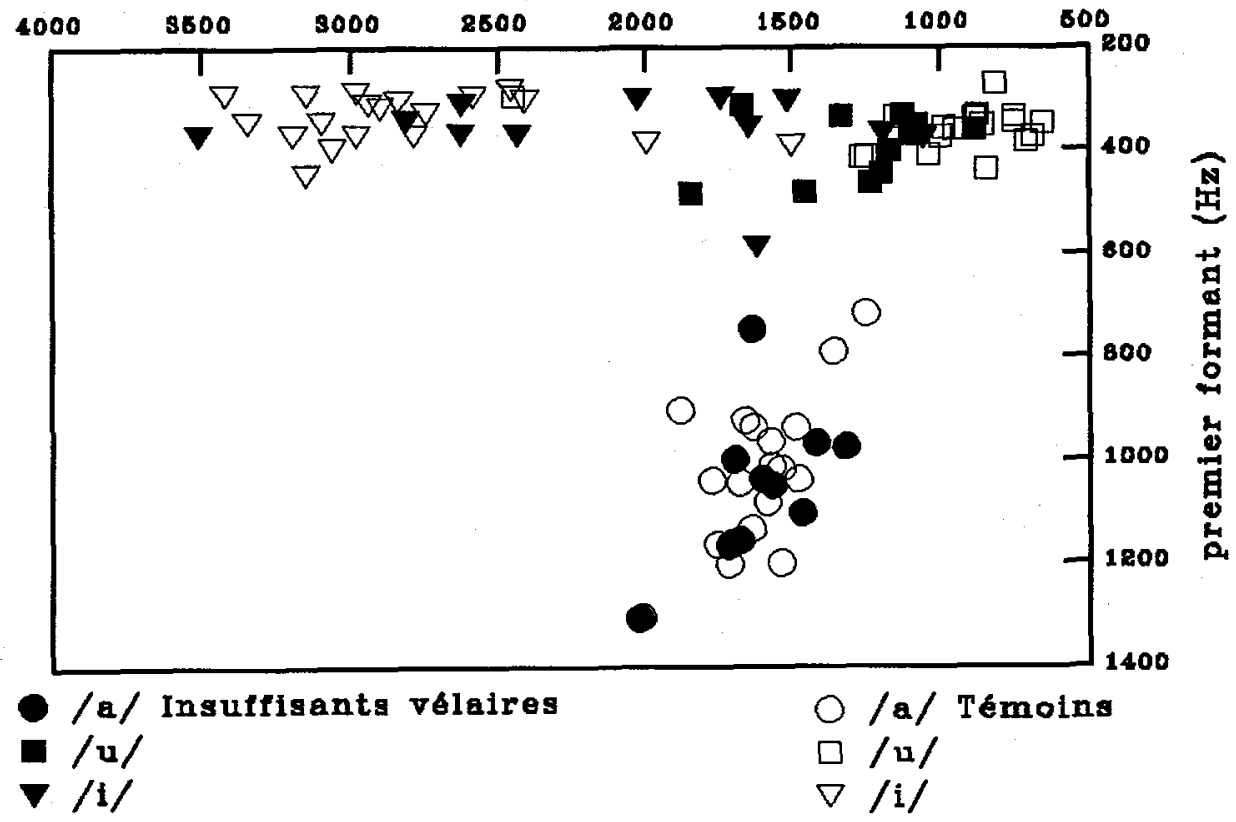

Figure 2 : Sujets dans le plan F1-F2. 